\title{
Résumé des recommandations sur la prévention du paludisme du Comité consultatif de la médecine tropicale et de la médecine des voyages (CCMTMV)
}

\author{
Boggild A. , Brophy J. ${ }^{2}$, Charlebois P. ${ }^{3}$, Crockett M. ${ }^{4}$, Geduld J. ${ }^{5}$, Ghesquiere W. ${ }^{6}$, McDonald P. ${ }^{7}$, \\ Plourde P. ${ }^{8}$, Teitelbaum P. ${ }^{9}$, Tepper $M .^{10}$, Schofield S. ${ }^{11}$ et McCarthy A. (présidente) ${ }^{12_{*}}$
}

1 University Health Network, Toronto General Hospital (Toronto, Ont.)

2 Division des maladies infectieuses, Centre hospitalier pour enfants de l'est de l'Ontario (Ottawa, Ont.)

3 Médecine interne, Centre des services de santé des Forces canadiennes (Atlantique) (Halifax, N.-É.)

4 Pédiatrie et santé des enfants, Université du Manitoba (Winnipeg, Man.)

5 Direction générale de la prévention et du contrôle des maladies infectieuses, Agence de la santé publique du Canada (Ottawa, Ont.)

6 Infectious Diseases and Internal Medicine, Université de la Colombie-Britannique (Victoria, C.-B.)

7 Direction des produits thérapeutiques, Santé Canada (Ottawa, Ont.)

8 Faculté de médecine, Université du Manitoba (Winnipeg, Man.)

9 Clinique santé-voyage Riverside (Ottawa, Ont.)

${ }^{10}$ Programme de lutte contre les maladies transmissibles, Direction de la protection de la santé de la Force (Ottawa, Ont.)

${ }^{11}$ Entomologie de la lutte antiparasitaire, Direction de la protection de la santé de la Force (Ottawa, Ont.)

${ }^{12}$ Clinique de médecine tropicale et de santé internationale, Division des maladies infectieuses, Campus général de l'Hôpital d'Ottawa (Ottawa, Ont.)

* Auteure-ressource : AMcCARTHY@Ottawahospital.on.ca

\section{Résumé}

Contexte : Au nom de l'Agence de la santé publique du Canada, le CCMTMV a formulé les Recommandations canadiennes pour la prévention et le traitement du paludisme (malaria) chez les voyageurs internationaux à l'intention des fournisseurs de soins de santé qui préparent les patients qui voyageront dans des régions impaludées et qui traitent les voyageurs qui sont revenus malades au pays.

Objectif : Donner des lignes directrices sur l'évaluation des risques et la prévention du paludisme.

Méthodologie : Le CCMTMV a examiné toutes les sources principales de renseignements sur la prévention du paludisme, de même que les recherches récentes et les données épidémiologiques nationales et internationales afin de proposer des lignes directrices adaptées au contexte canadien. II a formulé ses recommandations dans le cadre d'une approche de la médecine fondée sur les preuves à l'aide d'échelles d'évaluation afin de déterminer le poids et la qualité des preuves.

Recommandations : Utilisées en combinaison et de manière adéquate, les mesures de protection individuelle et la chimioprophylaxie se sont avérées très efficaces pour prévenir les infections au paludisme. Ces mesures comprennent la protection des lieux d'hébergement contre les moustiques, le port de vêtements adéquats, l'utilisation de moustiquaires imprégnées d'insecticides au préalable et l'application d'un insectifuge topique sur la peau exposée (contenant de 20 à $30 \%$ de DEET ou $20 \%$ d'icaridine). Pour choisir la chimioprophylaxie la plus appropriée, il faut examiner l'itinéraire du voyageur afin de déterminer son profil de risque de paludisme ainsi que les problèmes de pharmacorésistance potentiels. Au Canada, les antipaludéens disponibles sur ordonnance sont la chloroquine (ou hydroxychloroquine), l'atovaquone/proguanil, la doxycycline, la méfloquine et la primaquine. 


\section{Introduction}

Le paludisme est une maladie infectieuse grave causée par cinq différentes espèces de Plasmodium : $P$. falciparum, $P$. vivax, $P$. ovale, $P$. malariæ et $P$. knowlesi. Le paludisme est transmis par la morsure d'un moustique anophèle femelle infecté.

En 2009, $35 \%$ des voyageurs canadiens qui sont partis pour une destination autre que les États-Unis ont visité un pays où il existait un risque de contracter le paludisme, ce qui représente une augmentation de $131 \%$ par rapport à l'an 2000 (1,2). Entre les mois de septembre 2009 et septembre 2011, 94 cas de paludisme ont été diagnostiqués chez des voyageurs canadiens de retour au pays (3).

Selon les Centers for Disease Control and Prevention des États-Unis (CDC), le risque de contracter le paludisme pour les voyageurs américains (4-6) variait comme suit :

- Très élevé en Afrique occidentale et dans certaines régions de l'Océanie;

- Modéré dans les autres régions de l'Afrique, dans certaines régions de l'Amérique du Sud et en Asie du Sud;

- Faible dans la plupart des régions de l'Amérique centrale, dans les Caraïbes, au Mexique et dans d'autres régions d'Asie et d'Amérique du Sud;

- Minime dans les centres urbains de l'Asie du Sud-Est et de l'Amérique centrale et du Sud, ainsi que dans les grands centres de villégiature des Caraïbes et du Mexique.

Le Comité consultatif de la médecine tropicale et de la médecine des voyages (CCMTMV) donne de façon continue à l'Agence de la santé publique du Canada des conseils opportuns de nature médicale, scientifique et de santé publique concernant les maladies tropicales et les risques pour la santé associés aux voyages internationaux. II s'agit ici d'un résumé des Recommandations canadiennes pour la prévention et le traitement du paludisme (malaria) chez les voyageurs internationaux du CCMTMV, formulées à l'intention des fournisseurs de soins de santé qui préparent les patients qui voyageront dans des régions impaludées et qui traitent les voyageurs qui sont revenus malades au pays (7). Ces lignes directrices comprennent une description complète des recommandations sur l'évaluation des risques et la prévention du paludisme, une maladie qui demeure encore peu connue au Canada.

\section{Méthodologie}

Le sous-comité sur le paludisme, un groupe de travail du CCMTMV, a élaboré les lignes directrices. Chaque membre est bénévole, et aucun d'entre eux n'a signalé de conflit d'intérêts pertinent. Chaque chapitre a été mis à jour par un ou deux membres du sous-comité, puis examiné et approuvé par tous les membres du CCMTMV. La mise à jour a été effectuée en fonction d'un examen approfondi de la littérature. En outre, le sous-comité sur le paludisme a consulté toutes les principales sources d'information en matière de prévention et de traitement du paludisme, notamment l'Organisation mondiale de la Santé (OMS) (8), les Centers for Disease Control and Prevention (CDC) (6) des États-Unis et l'Advisory Committee on Malaria Prevention (Health Protection Agency) (9) du Royaume-Uni. Le sous-comité sur le paludisme a également examiné les recherches récentes ainsi que les données épidémiologiques nationales et internationales afin de proposer des recommandations adaptées au contexte canadien. Les facteurs déterminants comprennent l'homologation des médicaments, les habitudes de voyage propres aux Canadiens et l'épidémiologie du paludisme, ainsi que les valeurs et les préférences des voyageurs et des fournisseurs de soins de santé. Les recommandations dans le cadre d'une approche de la médecine fondée sur les preuves pour la prévention du paludisme ont été formulées à l'aide d'échelles d'évaluation afin de déterminer le poids et la qualité des preuves.

Le CCMTMV a pris en considération la nécessité de protection ainsi que les effets indésirables potentiels de la chimioprophylaxie. Les lignes directrices mettent également l'accent sur les divers degrés d'endémicité dans différentes régions. Le fournisseur de soins de santé doit être bien informé pour pouvoir conseiller chaque voyageur de manière adéquate. 


\section{Recommandations}

Les recommandations du CCMTMV dans le cadre d'une approche de la médecine fondée sur les preuves en ce qui concerne la prévention du paludisme sont résumées dans le Tableau 1. Une analyse de certaines recommandations principales suivra.

\section{Tableau 1 : Recommandations dans le cadre d'une approche de la médecine fondée sur les preuves en ce qui concerne la prévention du paludisme}

\section{Recommandation \\ 1. Utilisée de façon adéquate, la chimioprophylaxie du paludisme est très efficace (6). Catégorie MFP ${ }^{1}$ \\ 2. Les voyageurs devraient recevoir l'avis d'un spécialiste concernant les risques de paludisme et les stratégies à employer pour éviter les moustiques (10). \\ 3. Il est essentiel d'examiner soigneusement l'itinéraire d'un voyageur et ainsi déterminer le niveau d'endémicité et la durée de l'exposition pour pouvoir lui donner une évaluation précise des risques $(6,10,11)$. \\ 4. II est également important d'évaluer la santé et la tolérance aux risques des voyageurs avant de formuler des recommandations en matière de prévention du paludisme. \\ 5. Il est très important de suivre les recommandations en matière de prévention du paludisme (p. ex. la chimioprophylaxie et les mesures de protection individuelle) (12-22). \\ A I \\ B III \\ B III \\ B III \\ B III}

6. La chloroquine (Aralen ${ }^{\mathrm{MD}}$ ) ou I'hydroxychloroquine (Plaquenil ${ }^{\mathrm{MD}}$ ) sont les médicaments de choix pour les voyageurs qui se rendent dans des régions où les souches de paludisme sont sensibles à la chloroquine (23).

7. L'atovaquone/proguanil, la doxycycline ou la méfloquine sont des médicaments de choix pour les voyageurs qui se rendent dans des zones de résistance à la chloroquine ou de sensibilité à la méfloquine $(12-14,24-27)$.

8. L'atovaquone/proguanil et la doxycycline sont des médicaments de choix pour les voyageurs qui se rendent dans des zones où le paludisme est résistant à la méfloquine.

9. La primaquine est recommandée pour la chimioprophylaxie du paludisme chez les voyageurs qui ne veulent pas ou ne peuvent pas prendre de l'atovaquone/proguanil, de la doxycycline ou de la méfloquine dans des régions de résistance à la chloroquine.

10. Le traitement de réserve par l'atovaquone/proguanil ou la quinine et la doxycycline est recommandé pour les voyageurs qui ne peuvent obtenir un diagnostic dans les 24 heures.

11. La doxycycline est un antibiotique et ne devrait jamais être administrée en même temps qu'un vaccin oral vivant. L'administration de vaccins oraux vivants contre la typhoïde et le choléra doit être réalisée au moins trois jours avant la première dose de chloroquine, d'atovaquone/proguanil ou de méfloquine.

12. L'emploi concomitant de chloroquine nuit à la réponse immunitaire au vaccin antirabique cultivé sur les cellules diploïdes humaines et administré par voie intradermique. Si le vaccin intradermique contre la rage est administré à une personne qui prend de la chloroquine, il est recommandé d'effectuer un dosage des anticorps contre la rage après la vaccination pour vérifier si la réponse immunitaire est adéquate.

13. Utiliser des moustiquaires de lit imprégnées d'insecticide.

14. Appliquer un insectifuge topique sur les surfaces exposées de la peau pour se prémunir contre les morsures d'arthropodes et diminuer le risque d'exposition aux moustiques transmettant le paludisme.

15. Les produits homologués au Canada qui renferment de 20 à $30 \%$ de DEET (N,N-diéthyl-métatoluamide) ou $20 \%$ d'icaridine devraient être le premier choix des voyageurs canadiens.

16. Les insectifuges topiques qui contiennent du p-menthane-3,8-diol (une substance chimique provenant de l'eucalyptus à odeur de citronnelle) et qui sont homologués au Canada devraient

A I

A I

A I

A I

C III

B III

B III
A I

A I

A II

A II 


\section{Recommandation}

être considérés comme une deuxième option.

17. D'autres ingrédients actifs actuellement homologués au Canada comme insectifuges (p. ex. la citronnelle et l'huile de soya) ne sont pas disponibles partout ou n'offrent pas une protection d'une durée suffisante contre les piqûres ou les morsures. Leur utilisation n'est donc pas recommandée pour protéger les voyageurs contre les morsures de vecteurs.

18. Protéger les lieux de travail et d'hébergement contre les moustiques en installant des moustiquaires aux portes, aux fenêtres et aux corniches (l'espace ouvert entre le toit et le mur), en bouchant les trous dans les toits et les murs, et en cloisonnant les avant-toits et les espaces autour du bâtiment.

19. Porter des vêtements imprégnés d'insecticides.

20. Porter des vêtements appropriés (c'est-à-dire des vêtements pleine longueur, amples et de couleur claire, dérouler les manches et rentrer les extrémités du pantalon dans les bas ou les bottes).

21. II ne faut pas avoir recours ni se fier à des méthodes basées sur les insecticides telles que les spirales d'insecticides qui se brûlent, les insecticides en atomiseur, en aérosol et en pulvérisations spatiales, ainsi que les draps traités au moyen d'un insecticide.

22. Les mesures de protection individuelle qui sont inefficaces contre les arthropodes vecteurs et les maladies connexes, ou dont l'efficacité n'a pas été démontrée de façon convaincante ne sont pas recommandées. Citons en exemple les dispositifs électroniques (à ultrasons), les bracelets, colliers et bandes aux chevilles imprégnés d'insectifuge, les dispositifs d'électrocution (« bug zappers »), les pièges qui attirent les moustiques par l'odeur, le citrosa (plante d'intérieur ressemblant au géranium), la vitamine B1 administrée par voie orale et les hydratants pour la peau qui ne renferment pas d'ingrédients actifs à action insectifuge approuvés.

1 MFP = Médecine fondée sur les preuves. Les catégories MFP sont les suivantes : Force de la recommandation

A = Données suffisantes pour recommander l'utilisation

$\mathrm{B}=$ Données acceptables pour recommander l'utilisation

$\mathrm{C}=$ Données insuffisantes pour recommander ou déconseiller l'utilisation

$\mathrm{D}=$ Données acceptables pour déconseiller l'utilisation

$\mathrm{E}=$ Données suffisantes pour déconseiller l'utilisation

Qualité des preuves

I = Données obtenues dans le cadre d'au moins un essai comparatif convenablement randomisé

II = Données obtenues dans le cadre d'au moins un essai clinique bien conçu, sans randomisation, d'études de cohortes ou d'études analytiques cas-témoins, réalisés de préférence dans plus d'un centre, à partir de plusieurs séries chronologiques, ou résultats spectaculaires d'expériences non comparatives

III = Opinions exprimées par des sommités dans le domaine et reposant sur l'expérience clinique, des études descriptives ou des rapports de comités d'experts

\section{Évaluation des risques}

Le CCMTMV suggère un processus en deux volets pour évaluer les risques de paludisme : l'évaluation de l'exposition et l'évaluation de l'hôte.

Une évaluation de l'exposition permet de calculer la probabilité qu'un moustique infecté pique une personne. Elle tient compte des trois facteurs suivants :

- Les niveaux d'endémicité dans les zones de l'itinéraire de voyage;

- La présence ou la prédominance de $P$. falciparum;

- La durée de l'exposition.

Une évaluation de l'hôte permet de déterminer l'état de santé du voyageur lorsqu'il y a un risque potentiel de paludisme clinique ainsi que les indications relatives à l'administration de certains agents chimioprophylactiques antipaludéens, tout en tenant compte des préférences individuelles en ce qui concerne la gestion des risques. Parmi les facteurs à considérer, notons : 
- L'état de santé général du voyageur;

- Les interactions médicamenteuses;

- La probabilité d'avoir accès à des soins médicaux appropriés;

- La tolérance au risque et les préférences individuelles.

Une fois terminée, l'évaluation des risques peut éclairer la décision à savoir si une chimioprophylaxie antipaludéenne est recommandée et, le cas échéant, quel est l'agent chimioprophylactique indiqué :

- Si le risque de paludisme est minimal et que l'incidence de $P$. falciparum est nulle ou très basse, le CCMTMV recommande une chimioprophylaxie (en combinaison avec les mesures de protection individuelle) pour un séjour de plus de deux semaines.

- $\quad$ Si le risque de paludisme est minimal et que l'incidence de $P$. falciparum est élevée, le CCMTMV recommande une chimioprophylaxie (en combinaison avec les mesures de protection individuelle) pour un séjour de plus d'une semaine.

Les voyageurs qui décident de ne pas avoir recours à une chimioprophylaxie risquent davantage de contracter le paludisme, mais courent moins de risques de subir les effets indésirables des médicaments; l'inverse est vrai pour les personnes qui ont recours à une chimioprophylaxie.

Une caractérisation pays par pays des zones de transmission du paludisme se trouve dans la version complète des lignes directrices (7). L'Annexe fournit des recommandations sur le traitement chimioprophylaxique pour les 25 principales destinations présentant un risque de transmission du paludisme qui sont visitées par les Canadiens.

\section{Mesures de protection individuelle}

- Le risque qu'une personne soit piquée par un moustique peut être réduit grâce à l'utilisation de barrières physiques ou chimiques.

- Barrières physiques :

- Moustiquaires posées sur les portes, les fenêtres, les corniches et les autres espaces présents dans l'immeuble (28-30);

- Moustiquaires de lit imprégnées d'insecticide (31-33);

- Vêtements pleine longueur, amples et de couleur claire (les vêtements peuvent également être imprégnés d'insecticide).

- Les barrières chimiques repoussent ou tuent les moustiques $(34,35)$. Les principaux agents chimiques offerts sur le marché à l'heure actuelle sont les insectifuges topiques qu'on applique sur la peau exposée et les insecticides qui sont imprégnés dans les moustiquaires de lit et certains vêtements (36-40).

- Les insecticides topiques devraient contenir de 20 à $30 \%$ de DEET ou $20 \%$ d'icaridine.

- Par ailleurs, les insecticides topiques devant être considérés comme une deuxième option sont ceux qui contiennent du p-menthane-3,8-diol et qui sont homologués au Canada.

Les voyageurs devraient également être incités à prévoir leurs activités lors des périodes où les risques sont les plus faibles (p. ex. pendant la journée si les principaux vecteurs sont actifs en soirée) et à visiter des endroits où la transmission est la moins probable (p. ex. centres urbains, régions de hautes terres de plus de $2000 \mathrm{~m}$ ou $6500 \mathrm{pi})$.

\section{Chimioprophylaxie}

\section{Prescription de médicaments antipaludéens}

Prescrire une chimioprophylaxie antipaludéenne seulement après avoir effectué une évaluation des risques individuels. Pour obtenir des descriptions détaillées de la chimioprophylaxie et de la chimiothérapie, consulter le chapitre 8 des Recommandations canadiennes pour la prévention et le traitement du paludisme (malaria) chez 
les voyageurs internationaux (7). Le choix de l'agent chimioprophylactique le plus approprié s'effectue comme suit :

1. Examiner l'itinéraire de voyage précis du voyageur pour déterminer son profil de risque de paludisme.

2. Évaluer les avantages et les inconvénients des différents schémas thérapeutiques:

- Prendre en compte l'état de santé du voyageur, les autres médicaments que celui-ci prend ainsi que les risques d'effets indésirables et la nature de ceux-ci.

- Prendre en considération uniquement les médicaments les moins susceptibles d'exacerber un problème de santé ancien ou actuel.

3. Présenter au voyageur toutes les options possibles et, à moins qu'un médicament soit contre-indiqué, faiteslui choisir le schéma chimioprophylactique antipaludéen de première intention qu'il préfère.

4. Choisir la dose appropriée de médicament :

- Expliquer le schéma posologique à suivre, y compris la nécessité de prendre le médicament avant, pendant et après la visite de la zone à risque, préciser l'intérêt de prendre le médicament à la même heure chaque jour, informer le voyageur à savoir s'il faut prendre le médicament avec de la nourriture, puis aborder les précautions relatives aux effets secondaires de chaque médicament ( $p$. ex. l'exposition au soleil avec la doxycycline) (41-47).

5. Si le patient craint les effets indésirables potentiels d'un médicament, lui suggérer d'en faire l'essai.

6. Discuter avec le patient de la possibilité de changer de médicament pendant son voyage s'il éprouve alors des effets indésirables graves.

- Indiquer au voyageur que, si un médicament antipaludéen prescrit est bien toléré, il devrait continuer de le prendre, même s'il entend des rumeurs négatives à son sujet. Rien n'indique que l'utilisation à long terme d'agents chimioprophylactiques actuellement recommandés au Canada comporte un risque additionnel d'effets indésirables graves.

Discuter de l'importance de consulter rapidement un médecin si le voyageur se met à faire de la fièvre alors qu'il voyage dans une région impaludée ou dans l'année suivant son départ.

\section{Choix des antipaludéens pour certaines régions où le paludisme est pharmacorésistant} Avant de fournir des soins appropriés en vue d'un voyage, il faut consulter régulièrement les sources fiables (p. ex. Agence de la santé publique du Canada, CDC, ProMED) pour se tenir informé des nouveaux renseignements concernant les risques de paludisme. Cela s'applique particulièrement aux régions à risque minimal, car les changements peuvent avoir une incidence directe sur les recommandations à l'égard de la chimioprophylaxie. 
Tableau 2 : Choix des antipaludéens pour certaines régions où le paludisme est pharmacorésistant

\begin{tabular}{|c|c|}
\hline Zone/région $(6,48-50)$ & Médicaments de première intention \\
\hline $\begin{array}{l}\text { Régions où les souches sont sensibles à la } \\
\text { chloroquine : } \\
\text { Haïti, République dominicaine, Amérique centrale au } \\
\text { nord du canal de Panama, régions du Mexique, régions } \\
\text { de l'Amérique du Sud, de l'Afrique du Nord, régions du } \\
\text { Moyen-Orient, et Chine occidentale et centrale. }\end{array}$ & $\begin{array}{l}\text { Chloroquine (Aralen }{ }^{\mathrm{MD}} \text { ) } \\
\text { L'hydroxychloroquine (Plaquenil }^{\mathrm{MD}} \text { ) est une option } \\
\text { équivalente acceptable (51), à l'instar des trois } \\
\text { médicaments utilisés dans les zones de } \\
\text { chloroquinorésistance (voir ci-après). }\end{array}$ \\
\hline $\begin{array}{l}\text { Régions où les souches sont résistantes à la } \\
\text { chloroquine : } \\
\text { Majeure partie de l'Afrique subsaharienne, de l'Amérique } \\
\text { du Sud, de l'Océanie et de l'Asie. Voir ci-après pour } \\
\text { connaître les régions de résistance à la chloroquine et à } \\
\text { la méfloquine. }\end{array}$ & $\begin{array}{l}\text { Atovaquone/proguanil }(41,42,44-47,52) \\
\text { Doxycycline }(41,42,44-47,52) \\
\text { Méfloquine }(41,42,44-47,52)\end{array}$ \\
\hline $\begin{array}{l}\text { Régions où les souches sont résistantes à la } \\
\text { chloroquine et à la méfloquine : } \\
\text { Divers pays d'Asie, d'Afrique et du bassin de l'Amazone. } \\
\text { Toutefois, cela pose un véritable problème uniquement } \\
\text { dans les régions rurales et boisées situées le long de la } \\
\text { frontière thaïlandaise avec le Myanmar (Birmanie), le } \\
\text { Cambodge et le Laos, ainsi que dans le Vietnam du Sud. }\end{array}$ & $\begin{array}{l}\text { Atovaquone/proguanil }(44,53,54) \\
\text { Doxycycline }(44,53,54)\end{array}$ \\
\hline
\end{tabular}

Note : Voir l'Annexe « Risque de paludisme et chimioprophylaxie recommandée pour les 25 principales destinations de voyage visités par les Canadiens en 2012 et dans lesquelles le paludisme est endémique», ou une liste plus complète dans le document Recommandations canadiennes pour la prévention et le traitement du paludisme (malaria) chez les voyageurs internationaux (7).

\section{Arrêt des médicaments antipaludéens}

Des cas de décès dus au paludisme ont été recensés chez des voyageurs qui avaient délaissé toute chimioprophylaxie ou une chimioprophylaxie efficace au profit d'un autre traitement qui offrait une protection moindre $(24,51,55,56)$. II N'est PAS recommandé d'arrêter tout traitement chimioprophylactique.

Certains voyageurs ou prestataires de soins de santé pourraient suggérer de changer d'antipaludéen ou d'arrêter d'en prendre. La plupart du temps, il est conseillé d'ignorer ou de remettre en question de tels conseils. En effet, les médicaments employés dans d'autres régions du monde peuvent être moins efficaces, entraîner de graves effets indésirables et ne pas respecter les normes canadiennes. Citons par exemple le proguanil seul (Paludrine $^{\mathrm{MD}}$ ), la pyriméthamine (Daraprim ${ }^{\mathrm{MD}}$ ), les associations dapsone-pyriméthamine (Maloprim ${ }^{\mathrm{MD}}$ ) et méfloquine-sulfadoxine-pyriméthamine $\left(\right.$ Fansimef $\left.^{\mathrm{MD}}\right)$.

Cependant, si le voyageur souffre d'effets indésirables graves liés à l'agent chimioprophylactique, le médicament peut être changé, en particulier sur les conseils d'un fournisseur de soins de santé (de préférence celui qui a prescrit le médicament initial).

\section{Observance de la chimioprophylaxie}

Parmi les raisons de la non-observance figurent le fait de ne pas savoir que le paludisme est un risque, la crainte ou l'expérience passée d'effets secondaires des agents chimioprophylactiques, les fausses croyances en une immunité durable contre le paludisme qui aurait été acquise à la suite d'infections antérieures, le coût des médicaments et la confusion semée par des recommandations contradictoires. Cependant, peu d'information a été publiée sur les façons d'améliorer l'observance.

La non-observance et l'emploi sous-optimal de la chimioprophylaxie ou d'autres interventions préventives sont courants, plus particulièrement chez les grands randonneurs pédestres, les immigrants qui retournent dans leur 
pays d'origine, les voyageurs qui sont partis pour longtemps (plus d'un mois), les voyageurs de 40 ans ou moins et ceux qui doivent prendre des doses quotidiennes d'agents chimioprophylactiques $(12-21,23,25-27,57)$.

Quant aux prestataires de soins de santé, ils doivent être bien informés pour pouvoir bien conseiller les voyageurs (58). Les voyageurs qui font appel à une seule source d'information qualifiée (telle qu'un médecin de famille formé en médecine des voyages) sont beaucoup plus portés à suivre la prophylaxie antipaludéenne que ceux qui consultent une multitude de sources d'information possiblement contradictoires $(58,59)$.

\section{Résumé}

Le Tableau 3 résume les principaux changements apportés aux lignes directrices de 2014.

Tableau 3 : Résumé des principaux ajouts et changements aux lignes directrices de 2014 concernant la prévention du paludisme (7)

\section{Ajouts}

1. Une durée de séjour seuil a été ajoutée pour le traitement par chimioprophylaxie, de sorte que les fournisseurs de soins de santé puissent mieux adapter l'évaluation du risque individualisée (voir le chapitre 2).

2. Un nouvel insectifuge a été ajouté, l'icaridine à $20 \%$. Il est reconnu comme l'équivalent du DEET, comme choix de première intention contre les moustiques (voir le chapitre 3 ).

3. Les lignes directrices ont été étendues aux sous-populations nécessitant une attention spéciale, c'est-à-dire les enfants, les migrants, les expatriés et les voyageurs visitant des amis et de la famille, les femmes enceintes ou qui allaitent, et les voyageurs souffrant de comorbidités (voir le chapitre 5).

4. Une nouvelle fiche sur le paludisme peut être donnée aux voyageurs pour leur fournir des renseignements sur leur chimioprophylaxie antipaludéenne tout en leur rappelant de consulter un médecin en cas de fièvre après un voyage.

\section{Changements}

1. Le chapitre 4, "Prévention - Régimes chimioprophylactiques », a été peaufiné afin qu'il soit plus facile de comprendre les choix de médicaments à disposition. Ces modifications comprennent : une approche simplifiée et par étapes pour choisir la prophylaxie antipaludéenne; une liste exhaustive des médicaments et des risques de paludisme par pays/région, présentée sous forme de tableaux; un approfondissement de l'explication des différences entre les approches en matière de prophylaxie antipaludéenne dans d'autres territoires.

2. Le chapitre 8, "Médicaments pour la prévention et le traitement du paludisme ", comprend une mise à jour sur l'utilisation de la primaquine pour la prophylaxie et la prévention du paludisme, des renseignements actualisés sur la posologie pour les enfants de l'atovaquone et du proguanil ainsi que des mises à jour générales au tableau 8.11: Médicaments (nom générique et commercial) pour la prévention et le traitement du paludisme. Des révisions ont également été apportées aux sous-sections relatives à la prévention du paludisme : chloroquine et méfloquine (soulignant davantage le choix ou le rejet de ce médicament en fonction de la tolérance de chacun).

\section{Remerciements}

Le CCMTMV tient à remercier Joanna Odrowaz et Elspeth Payne pour leur contribution à l'élaboration des sommaires et Manisha Kulkarni pour sa contribution à la déclaration.

Membres du CCMTMV : Boggild A., Brophy J., Bui Y. G., Crockett M., Ghesquiere W., Greenaway C., Henteleff A., Libman M., Teitelbaum P. et McCarthy A. (présidente).

Représentants chargés de la liaison : Hui C. (Société canadienne de pédiatrie) et Gershman M. (Centers for Disease Control and Prevention [É.-U.]).

Membres d'office : Marion D. (Centre des services de santé des Forces canadiennes, ministère de la Défense nationale), McDonald P. (Division des médicaments anti-infectieux, Santé Canada), Schofield S. (Direction de la 
protection de la santé de la Force, ministère de la Défense nationale) et Tepper M. (Direction de la protection de la santé de la Force, ministère de la Défense nationale).

Membre émérite : Jeanes C. W. L.

\section{Conflit d'intérêts}

II n'y a aucun conflit d'intérêts à déclarer.

\section{Financement}

Ce travail a été appuyé par l'Agence de la santé publique du Canada.

\section{Références}

(1) Organisation mondiale de la Santé. World Malaria Report 2012. 2012:1-195.

(2) Geduld J, Bryson M et Straight-Caron T. Canadian Trends of International Travel and Risk of Malaria Exposure, 12th Conference of the International Society of Travel Medicine, 8-12 mai 2011, Boston, É.-U., 2011.

(3) Boggild A, Geduld J, Libman M, Ward B, McCarthy A, Doyle P, Ghesquiere W, Vincelette J, Kuhn S, Freedman $\mathrm{D}$ et Kain K. Travel-acquired infections and illnesses in Canadians: surveillance report from CanTravNet surveillance data, 2009-2011. Open Med 2014;8(1).

(4) Mali S, Tan KR et Arguin PM. Division of Parasitic Diseases and Malaria, Center for Global Health. Centers for Disease Control and Prevention. Malaria surveillance - United States, 2009. MMWR Surveill Summ. Avril 2011;60(3):1-15.

(5) Mali S, Steele S, Slutsker L et Arguin P. Malaria surveillance - United States, 2008. MMWR 2010;59(7):1-15.

(6) Centers for Disease Control and Prevention (CDC). CDC Health Information for International Travel 2012. New York: Oxford University Press; 2012.

(7) Committee to Advise on Tropical Medicine and Travel. Canadian Recommendations for the Prevention and Treatment of Malaria (sous presse). Accès : www.publications.gc.ca

(8) Organisation mondiale de la Santé. Voyages internationaux et santé. Genève (Suisse) : Organisation mondiale de la Santé; 2012.

(9) Bradley D et Bannister B, Health Protection Agency Advisory Committee on Malaria Prevention for UK Travellers. Guidelines for malaria prevention in travellers from the United Kingdom for 2003. Commun Dis Public Health 2003;6(3):180-199.

(10) Steffen R, deBernardis C et Banos A. Travel epidemiology - a global perspective. Int J Antimicrob Agents 2003;21(2):89-95.

(11) Leder K, Black J, O'Brien D, Greenwood Z, Kain KC, Schwartz E et al. Malaria in travelers: a review of the GeoSentinel surveillance network. Clin Infect Dis. Oct. 2004;39(8):1104-1112.

(12) Chatterjee S. Compliance of malaria chemoprophylaxis among travelers to India. J Travel Med. Mars 1999;6(1):7-11.

(13) Laver SM, Wetzels J et Behrens RH. Knowledge of malaria, risk perception, and compliance with prophylaxis and personal and environmental preventive measures in travelers exiting Zimbabwe from Harare and Victoria Falls International airport. J Travel Med. Nov.-déc. 2001;8(6):298-303.

(14) Banerjee D et Stanley PJ. Malaria chemoprophylaxis in UK general practitioners traveling to South Asia. $J$ Travel Med. Juil.-août 2001;8(4):173-175.

(15) Lobel HO, Baker MA, Gras FA, Stennies GM, Meerburg P, Hiemstra E et al. Use of malaria prevention measures by North American and European travelers to East Africa. J Travel Med. Juil.-août 2001;8(4):167172. 
(16) Leonard L et VanLandingham M. Adherence to travel health guidelines: the experience of Nigerian immigrants in Houston, Texas. J Immigr Health. Jan. 2001;3(1):31-45.

(17) Morgan M et Figueroa-Munoz JI. Barriers to uptake and adherence with malaria prophylaxis by the African community in London, England: focus group study. Ethn Health. Nov. 2005;10(4):355-372.

(18) Alon D, Shitrit $P$ et Chowers M. Risk behaviors and spectrum of diseases among elderly travelers: a comparison of younger and older adults. J Travel Med. Juil.-août 2010;17(4):250-255.

(19) Toovey S, Moerman F et van Gompel A. Special infectious disease risks of expatriates and long-term travelers in tropical countries. Part I: malaria. J Travel Med. Jan.-févr. 2007;14(1):42-49.

(20) Baggett HC, Graham S, Kozarsky PE, Gallagher N, Blumensaadt S, Bateman J et al. Pretravel health preparation among US residents traveling to India to VFRs: importance of ethnicity in defining VFRs. $J$ Travel Med. Mars-avril 2009;16(2):112-118.

(21) Piyaphanee W, Wattanagoon Y, Silachamroon U, Mansanguan C, Wichianprasat P et Walker E. Knowledge, attitudes, and practices among foreign backpackers toward malaria risk in southeast Asia. $J$ Travel Med. Mars-avril 2009;16(2):101-106.

(22) Abraham C, Clift S et Grabowski P. Cognitive predictors of adherence to malaria prophylaxis regimens on return from a malarious region: a prospective study. Soc Sci Med 1999;48(11):1641-54.

(23) Queyriaux B, Texier G, Ollivier L, Galoisy-Guibal L, Michel R, Meynard JB et al. Plasmodium vivax malaria among military personnel, French Guiana, 1998-2008. Emerg Infect Dis. Juillet 2011;17(7):1280-1282.

(24) Kain KC, MacPherson DW, Kelton T, Keystone JS, Mendelson J et MacLean JD. Malaria deaths in visitors to Canada and in Canadian travellers: a case series. CMAJ. Mars 2001;164(5):654-659.

(25) Landry P, lorillo D, Darioli R, Burnier M et Genton B. Do travelers really take their mefloquine malaria chemoprophylaxis? Estimation of adherence by an electronic pillbox. J Travel Med. Jan.-févr. 2006;13(1):814.

(26) Molle I, Christensen KL, Hansen PS, Dragsted UB, Aarup M et Buhl MR. Use of medical chemoprophylaxis and antimosquito precautions in Danish malaria patients and their traveling companions. J Travel Med. Sept.oct. 2000;7(5):253-258.

(27) Ollivier L, Michel R, Carlotti MP, Mahe P, Romand O, Todesco A et al. Chemoprophylaxis compliance in a French battalion after returning from malaria-endemic area. J Travel Med. Sept.-oct. 2008;15(5):355-357.

(28) Lindsay SW, Jawara M, Paine K, Pinder M, Walraven GEL et Emerson PM. Changes in house design reduce exposure to malaria mosquitoes. Trop Med Int Health 2003;8(6):512-517.

(29) Lindsay SW, Emerson PM et Charlwood JD. Reducing malaria by mosquito-proofing houses. Trends Parisitol 2002;18(11):510-514.

(30) Njie M, Dilger E, Lindsay S et Kirby M. Importance of eaves to house entry by anopheline, but not culicine, mosquitoes. J Med Entomol. 2009;46(3):505-10.

(31) Christophers SR. Mosquito repellents; being a report of the work of the Mosquito Repellent Inquiry, Cambridge, 1943-5. J Hyg. 1947;45(2):176-231.

(32) Schoepke A, Steffen R et Gratz N. Effectiveness of personal protection measures against mosquito bites for malaria prophylaxis in travelers. J Travel Med. 1998;5(4):188-192.

(33) Joy RJT. Malaria in American troops in the South and Southwest Pacific in World War II. Med Hist 1999;43(02):192-207.

(34) Maia M et Moore S. Plant-based insect repellents: a review of their efficacy, development and testing. Malar J 2011;10:S11.

(35) Moore SJ et Debboun M. History of insect repellents. In: Debboun M, Francis S et Strickman DA, directeurs. Insect repellents: principles, methods and uses. Première édition: CRC Press; 2006. p. 3-29.

(36) Lengeler C. Insecticide-treated bed nets and curtains for preventing malaria. Cochrane Database Syst Rev 2004;2(CD000363). 
(37) Schreck CE, Posey K et Smith D. Durability of permethrin as a potential clothing treatment to protect against blood-feeding arthropods. J Econ Entomol 1978;71(3):397-400.

(38) Schreck CE, Haile DG et Kline DL. The effectiveness of permethrin and deet, alone or in combination, for protection against Aedes taeniorhynchus. Am J Trop Med Hyg 1984;33(4):725-730.

(39) Vaughn MF et Meshnick SR. Pilot study assessing the effectiveness of long-lasting permethrin-impregnated clothing for the prevention of tick bites. Vector Borne Zoonotic Dis 2011;11(7):869-875.

(40) Soto J, Medina F, Dember N et Berman J. Efficacy of permethrin-impregnated uniforms in the prevention of malaria and leishmaniasis in Colombian soldiers. Clin Infect Dis. Sept. 1995;21(3):599-602.

(41) Shanks GD, Kremsner PG, Sukwa TY, Van Der Berg JD, Shapiro TA, Scott TR et al. Atovaquone and proguanil hydrochloride for prophylaxis of malaria. J Travel Med. 1999;6(Suppl 1):S21-S27.

(42) Sanchez J, DeFraites R, Sharp T et Hanson R. Mefloquine or doxycycline prophylaxis in US troops in Somalia. Lancet 1993;341(8851):1021-2.

(43) Koren G, Matsui D et Bailey B. DEET-based insect repellents: safety implications for children and pregnant and lactating women. CMAJ 2003;169(3):209-12.

(44) Ohrt C, Richie T, Widjaja H, Shanks G, Fitriadi J, Fryauff D et al. Mefloquine compared with doxycycline for the prophylaxis of malaria in Indonesian soldiers. A randomized, double-blind, placebo-controlled trial. Ann Intern Med 1997;126(12):963-72.

(45) Weiss W, Oloo A, Johnson A, Koech D et Hoffman S. Daily primaquine is effective for prophylaxis against falciparum malaria in Kenya: comparison with mefloquine, doxycycline, and chloroquine plus proguanil. $J$ Infect Dis 1995;171(6):1569-75.

(46) Sukwa T, Mulenga M, Chisdaka N, Roskell N et Scott T. A randomized, double-blind, placebo-controlled field trial to determine the efficacy and safety of Malarone (atovaquone/proguanil) for the prophylaxis of malaria in Zambia. Am J Trop Med Hyg 1999;60(4):521-5.

(47) Shanks G, Gordon D, Klotz F, Aleman G, Oloo A, Sadie D et al. Efficacy and safety of atovaquone/proguanil as suppressive prophylaxis for Plasmodium falciparum malaria. Clin Infect Dis 1998;27(3):494-9.

(48) Centers for Disease Control and Prevention (CDC). CDC Health Information for International Travel 2014. New York: Oxford University Press; 2013.

(49) Wongsrichanalai C, Sirichaisinthop J, Karwacki JJ, Congpuong K, Miller RS, Pang L et al. Drug resistant malaria on the Thai-Myanmar and Thai-Cambodian borders. Southeast Asian J Trop Med Public Health. Mars 2001;32(1):41-49.

(50) Wongsrichanalai C, Pickard AL, Wernsdorfer WH et Meshnick SR. Epidemiology of drug-resistant malaria. Lancet Infect Dis. Avril 2002;2(4):209-218.

(51) Newman R, Parise M, Barber A et Steketee R. Malaria-related deaths among U.S. travelers, 1963-2001. Ann Intern Med 2004;141(7):547-55.

(52) Lell B, Luckner D, Ndjave M, Scott T et Kremsner P. Randomised placebo-controlled study of atovaquone plus proguanil for malaria prophylaxis in children. Lancet 1998;351(9104):709-13.

(53) Camus D, Djossou F, Schilthuis HJ, Hogh B, Dutoit E, Malvy D et al. Atovaquone-proguanil versus chloroquine-proguanil for malaria prophylaxis in nonimmune pediatric travelers: results of an international, randomized, open-label study. Clin Infect Dis. Juin 2004;38(12):1716-1723.

(54) Krudsood S, Patel SN, Tangpukdee N, Thanachartwet W, Leowattana W, Pornpininworakij K et al. Efficacy of atovaquone-proguanil for treatment of acute multidrug-resistant Plasmodium falciparum malaria in Thailand. Am J Trop Med Hyg. Avril 2007;76(4):655-658.

(55) Humar A, Sharma S, Zoutman D et Kain KC. Fatal falciparum malaria in Canadian travellers. CMAJ. Avril 1997;156(8):1165-1167.

(56) Centers for Disease Control and Prevention (CDC). Malaria deaths following inappropriate malaria chemoprophylaxis - United States, 2001. MMWR Morb Mortal Wkly Rep. Juil. 2001;50(28):597-599. 
(57) Pistone T, Ezzedine K, Gaudin AF, Hercberg S, Nachbaur G et Malvy D. Malaria prevention behaviour and risk awareness in French adult travellers. Travel Med Infect Dis. Janv. 2010;8(1):13-21.

(58) Ropers G, Du Ry van Beest Holle M, Wichmann O, Kappelmayer L, Stuben U, Schonfeld C et al. Determinants of malaria prophylaxis among German travelers to Kenya, Senegal, and Thailand. J Travel Med. Mai-juin 2008;15(3):162-171.

(59) Held TK, Weinke T, Mansmann U, Trautmann M et Pohle HD. Malaria prophylaxis: identifying risk groups for non-compliance. Q J Med. Janv. 1994;87(1):17-22.

(60) Institut de médecine sociale et préventive de l'Université de Zurich. Santé-voyages: Vaccinations et mesures antipaludiques 2010. 2010. Accès :

http://www.bag.admin.ch/themen/medizin/00682/00685/03062/index.html?lang=fr [consulté le 18 novembre 2010].

(61) Organisation mondiale de la Santé. Voyages internationaux et santé, Liste par pays. 2011. Accès : http://www.who.int/ith/fr/

(62) Deutsche Gesellschaft für Tropenmedizin und Internationale Gesundheit (DTG). Empfehlungen zur Malariavorbeugung. 2011. Accès : http://www.dtg.org/uploads/media/Malaria_2011.pdf [consulté le 12 juin 2011].

(63) Office fédéral de la santé publique, Confédération suisse, Division maladies transmissibles. Paludisme (malaria) - mise à jour 2010. OFSP 2010;19:506-8.

(64) Smittskyddsinstitutet. Rekommendationer för malariaprofylax 2010. 2010. Accès :

http://www.folkhalsomyndigheten.se/publicerat-material/publikationer/?topic=smittskydd-och-sjukdomar [consulté le 24 novembre 2010].

(65) International Association for Medical Assistance to Travellers 2011. World Malaria Risk Chart. 2011. Accès : http://www.iamat.org/disease_details.cfm?id=140

(66) Haut conseil de la santé publique. Recommandations sanitaires pour les voyageurs 2011 (à l'intention des professionnels de la santé). 2011. Accès : http://opac.invs.sante.fr/doc_num.php?explnum_id=7068 [consulté le $1^{\text {er }}$ juin 2011].

(67) Statistique Canada. Enquête sur les voyages internationaux : Résidents canadiens 2012 (extrait fait sur mesure). 


\section{Annexe}

Appendice : Risque de paludisme et chimioprophylaxie recommandée pour les 25 principales destinations de voyage visités par les Canadiens en 2012 et dans lesquelles le paludisme est endémique (6, 60-67).

\begin{tabular}{|c|c|c|c|c|c|}
\hline \multicolumn{2}{|r|}{ Pays } & \multirow[b]{2}{*}{$\begin{array}{l}\text { Régions de transmission du paludisme } \\
\text { Transmission du paludisme minimale } \\
\text { ou absente dans les principales régions } \\
\text { de villégiature sur le littoral, y compris } \\
\text { la ville d'Acapulco ou le long de la } \\
\text { Riviera Maya, y compris les villes de } \\
\text { Cancún, de Cozumel et de Playa del } \\
\text { Carmen. Aucune transmission le long } \\
\text { de la frontière avec les États-Unis. } \\
\\
\text { Peu de transmission du paludisme } \\
\text { dans les États de Jalisco, de Quintana } \\
\text { Roo, de Sonora et de Tabasco. }\end{array}$} & \multirow{2}{*}{$\begin{array}{l}\text { Chimioprophylaxie } \\
\text { recommandée par le CCMTMV* } \\
(2-9)\end{array}$} & \multirow{4}{*}{$\begin{array}{l}\text { Saison }{ }^{(3 ; 4)} \\
\text { À l'année }\end{array}$} & \multirow{4}{*}{$\begin{array}{l}\text { Plasmodium } \\
\text { falciparum }^{(2-4)} \\
\text { (en } \%) \\
\end{array}$} \\
\hline \multirow[t]{3}{*}{1} & \multirow[t]{3}{*}{ Mexique } & & & & \\
\hline & & $\begin{array}{l}\text { Risque modéré dans certaines parties } \\
\text { des États de Chiapas et de Oaxaca. }\end{array}$ & Chloroquine. & & \\
\hline & & $\begin{array}{l}\text { Risque faible dans les régions rurales } \\
\text { des États de Nayarit, de Sinaloa, de } \\
\text { Chihuahua et de Durango. }\end{array}$ & $\begin{array}{l}\text { Chloroquine pour les séjours } \\
>1 \text { semaine; chloroquine ou } \\
\text { mesures de protection } \\
\text { individuelle uniquement pour les } \\
\text { séjours } \leq 1 \text { semaine. }\end{array}$ & & \\
\hline \multirow[t]{3}{*}{2} & \multirow[t]{3}{*}{$\begin{array}{l}\text { République } \\
\text { dominicaine }\end{array}$} & $\begin{array}{l}\text { Peu, voire aucune transmission du } \\
\text { paludisme dans les zones de } \\
\text { villégiature de Romana et de Samaná, } \\
\text { ou dans les villes de Santo Domingo, } \\
\text { de Santiago et de Puerto Plata. }\end{array}$ & $\begin{array}{l}\text { Aucune; utiliser des mesures de } \\
\text { protection individuelle. }\end{array}$ & \multirow{3}{*}{ À l'année } & \multirow{3}{*}{100} \\
\hline & & $\begin{array}{l}\text { Certaines transmissions ont eu lieu par } \\
\text { le passé dans la province de La } \\
\text { Altagracia, y compris dans les zones de } \\
\text { villégiature comme Punta Cana. }\end{array}$ & $\begin{array}{l}\text { En l'absence de toute éclosion } \\
\text { récente dans la province de La } \\
\text { Altagracia, des mesures de } \\
\text { protection individuelles } \\
\text { uniquement sont } \\
\text { recommandées pour les centres } \\
\text { de villégiature de cette province. } \\
\text { Consulter un médecin en cas de } \\
\text { fièvre. }\end{array}$ & & \\
\hline & & $\begin{array}{l}\text { Zones rurales, avec un risque élevé } \\
\text { dans les provinces de Dajabón, d'Elias } \\
\text { Piña et de San Juan, à la frontière } \\
\text { d'Haïti. }\end{array}$ & Chloroquine. & & \\
\hline \multirow[t]{3}{*}{3} & \multirow[t]{3}{*}{ Chine } & $\begin{array}{l}\text { Aucune transmission du paludisme } \\
\text { dans les zones urbaines ou dans le } \\
\text { nord de la Chine. }\end{array}$ & Aucune. & S.O. & S.O. \\
\hline & & $\begin{array}{l}\text { Transmission limitée du paludisme à } P \text {. } \\
\text { vivax dans les provinces du sud et } \\
\text { dans certaines provinces du centre, y } \\
\text { compris Anhui, Ghuizhou, Henan, } \\
\text { Hubei et Jiangsu. } \\
\text { Le risque de contracter le paludisme } \\
\text { dans le centre de la Chine est faible. }\end{array}$ & $\begin{array}{l}\text { Pour les voyageurs se rendant } \\
\text { dans les grandes villes et } \\
\text { faisant des excursions d'une } \\
\text { journée à la campagne ou sur le } \\
\text { fleuve Yangtze : aucune; utiliser } \\
\text { des mesures de protection } \\
\text { individuelle. } \\
\text { Pour les personnes voyageant } \\
\text { beaucoup dans ou à travers les } \\
\text { régions rurales du sud de la } \\
\text { Chine: chloroquine. }\end{array}$ & \multirow[t]{2}{*}{ À l'année } & \multirow[t]{2}{*}{9} \\
\hline & & $\begin{array}{l}\text { La transmission du paludisme à } \\
P . f a l c i p a r u m \text { se produit dans la } \\
\text { province de Yunnan et, dans une } \\
\text { moindre mesure, dans la province de } \\
\text { Hainan. Des résistances de } P \text {. } \\
\text { falciparum à la chloroquine et à la } \\
\text { pyriméthamine-sulfadoxine ont été } \\
\text { signalées. }\end{array}$ & ATQ-PG, DOXY ou MFQ. & & \\
\hline
\end{tabular}




\begin{tabular}{|c|c|c|c|c|c|}
\hline & & $\begin{array}{l}\text { Une résistance de } P \text {. falciparum à la } \\
\text { méfloquine a été signalée dans la } \\
\text { province de Yunnan, dans les régions } \\
\text { frontalières de la Birmanie (Myanmar). }\end{array}$ & ATQ-PG ou DOXY. & & \\
\hline \multirow[t]{2}{*}{4} & \multirow[t]{2}{*}{ Inde } & $\begin{array}{l}\text { Aucune transmission du paludisme à } \\
\text { plus de } 2000 \text { m d'altitude dans } \\
\text { certaines parties des États d'Himachal } \\
\text { Pradesh, de Jammu-Cachemire et de } \\
\text { Sikkim. }\end{array}$ & Aucune. & S.O. & S.O. \\
\hline & & $\begin{array}{l}\text { Toutes les autres régions, y compris la } \\
\text { plupart des zones urbaines comme } \\
\text { Bombay (Mumbai) et Delhi. } \\
\text { Le risque est plus faible dans la plupart } \\
\text { des régions les plus au sud de l'Inde. } \\
\text { Le risque est faible dans les régions } \\
\text { urbaines centrales d'Agra et de } \\
\text { Bangalore. }\end{array}$ & $\begin{array}{l}\text { ATQ-PG, DOXY ou MFQ } \\
\text { Des mesures de protection } \\
\text { individuelle peuvent être } \\
\text { envisagées pour les séjours < } 1 \\
\text { semaine dans les régions } \\
\text { urbaines centrales de Delhi, } \\
\text { d'Agra et de Bangalore. }\end{array}$ & À l'année & $>40$ \\
\hline \multirow[t]{2}{*}{5} & \multirow[t]{2}{*}{ Costa Rica } & $\begin{array}{l}\text { Peu de risque, voire aucun risque de } \\
\text { transmission du paludisme dans une } \\
\text { grande partie du pays, à l'exception des } \\
\text { régions indiquées ci-dessous. Aucune } \\
\text { transmission du paludisme dans la ville } \\
\text { de Limón (Puerto Limón). }\end{array}$ & $\begin{array}{l}\text { Aucune; utiliser des mesures de } \\
\text { protection individuelle. }\end{array}$ & \multirow[t]{2}{*}{ À l'année } & \multirow[t]{2}{*}{$\begin{array}{l}\text { Principalemen } \\
\text { P. vivax }\end{array}$} \\
\hline & & $\begin{array}{l}\text { Province de Limón (sauf la ville de } \\
\text { Limón), principalement dans le canton } \\
\text { de Matina. }\end{array}$ & Chloroquine. & & \\
\hline \multirow[t]{2}{*}{6} & \multirow[t]{2}{*}{ Thaillande } & $\begin{array}{l}\text { Aucune transmission du paludisme } \\
\text { dans les villes, y compris Bangkok, } \\
\text { Chiang Mai, Chiang Rai, Pattaya, Koh } \\
\text { Samui, Phang Nga, Phuket (ville) et } \\
\text { Koh Phangan, ou dans les principaux } \\
\text { centres de villégiature touristiques. }\end{array}$ & Aucune. & S.O. & S.O. \\
\hline & & $\begin{array}{l}\text { Régions forestières rurales à proximité } \\
\text { des frontières avec le Cambodge, la } \\
\text { Birmanie (Myanmar) et le Laos. } \\
\text { Régions forestières dans les districts } \\
\text { de Phang Nga et de Phuket. Certaines } \\
\text { îles présentent un risque de } \\
\text { transmission du paludisme. } \\
\text { Une résistance à la méfloquine a été } \\
\text { signalée. }\end{array}$ & ATQ-PG ou DOXY. & À l'année & $50-75$ \\
\hline \multirow[t]{2}{*}{7} & \multirow[t]{2}{*}{ Philippines } & $\begin{array}{l}\text { Peu de transmission, voire aucune } \\
\text { transmission du paludisme dans les } \\
\text { zones urbaines ou sur les îles qui ne } \\
\text { sont pas énumérées ci-dessous. }\end{array}$ & $\begin{array}{l}\text { Aucune; utiliser des mesures de } \\
\text { protection individuelle. }\end{array}$ & \multirow{2}{*}{ À l'année } & \multirow{2}{*}{$70-80$} \\
\hline & & $\begin{array}{l}\text { Régions rurales jusqu'à } 600 \mathrm{~m} \\
\text { d'altitude sur les îles suivantes : Basilu, } \\
\text { Luzon, Mindanao, Mindoro, Palawan, } \\
\text { Sulu (Jolo) et Tawi-Tawi. }\end{array}$ & ATQ-PG, DOXY ou MFQ. & & \\
\hline \multirow[t]{2}{*}{8} & \multirow[t]{2}{*}{$\begin{array}{l}\text { Afrique du } \\
\text { Sud }\end{array}$} & $\begin{array}{l}\text { Aucune transmission du paludisme } \\
\text { dans la majeure partie du pays, y } \\
\text { compris sur la Garden Route et dans } \\
\text { les principales villes. }\end{array}$ & Aucune. & S.O. & S.O. \\
\hline & & $\begin{array}{l}\text { Régions à basse altitude dans les } \\
\text { provinces de Mpumalanga (y compris } \\
\text { le parc national Kruger), de Limpopo } \\
\text { (anciennement Northern) et dans le } \\
\text { nord-est de la province Kwa Zulu-Natal } \\
\text { jusqu'au fleuve Tugela, au sud. }\end{array}$ & ATQ-PG, DOXY ou MFQ. & $\begin{array}{l}\text { À l'année } \\
\text { (le risque } \\
\text { est au plus } \\
\text { haut } \\
\text { d'octobre à } \\
\text { mai) }\end{array}$ & 90 \\
\hline 9 & Pérou & $\begin{array}{l}\text { Aucun risque de paludisme à plus de } \\
2000 \mathrm{~m} \text { d'altitude, y compris les } \\
\text { régions des hautes terres touristiques } \\
\text { (Machu Picchu, lac Titicaca et les villes }\end{array}$ & Aucune. & S.O. & S.O. \\
\hline
\end{tabular}




\begin{tabular}{|c|c|c|c|c|c|}
\hline & & $\begin{array}{l}\text { d'Arequipa, de Cuzco et de Puno) ou } \\
\text { dans les villes de Lima et au sud de } \\
\text { Lima, y compris Moquegua, Nazca et } \\
\text { Tacna. }\end{array}$ & & & \\
\hline & & $\begin{array}{l}\text { Toutes les régions jusqu'à } 2000 \text { m (à } \\
\text { l'exception des villes susmentionnées). } \\
\text { Cela comprend les villes de Puerto } \\
\text { Maldonado et d'lquitos. La plupart des } \\
\text { cas de } P \text {. Falciparum se produisent } \\
\text { dans la région de Loreto. }\end{array}$ & ATQ-PG, DOXY ou MFQ. & À l'année & 15 \\
\hline \multirow[t]{2}{*}{10} & \multirow[t]{2}{*}{ Turquie } & $\begin{array}{l}\text { Aucune transmission du paludisme } \\
\text { dans les régions ouest et nord-est du } \\
\text { pays, y compris les destinations } \\
\text { touristiques communes que } \\
\text { représentent les villes d'Izmir et } \\
\text { d'Istanbul, et la région de Cappadoce. }\end{array}$ & Aucune. & S.O. & S.O. \\
\hline & & $\begin{array}{l}\text { Transmission limitée du paludisme } \\
\text { dans le sud-est du pays. }\end{array}$ & $\begin{array}{l}\text { Chloroquine pour les séjours } \\
>2 \text { semaines; chloroquine ou } \\
\text { mesures de protection } \\
\text { individuelle uniquement pour les } \\
\text { séjours } \leq 2 \text { semaines. }\end{array}$ & $\begin{array}{l}\text { Mai à } \\
\text { octobre }\end{array}$ & Sporadiquement \\
\hline \multirow[t]{3}{*}{11} & \multirow[t]{3}{*}{ Argentine } & $\begin{array}{l}\text { Aucune transmission du paludisme } \\
\text { dans les régions urbaines, les chutes } \\
\text { d'Iguazu ou dans les provinces qui ne } \\
\text { sont pas énumérées ci-dessous. }\end{array}$ & Aucune. & S.O. & S.O. \\
\hline & & $\begin{array}{l}\text { Rare dans la province de Misiones, le } \\
\text { long de la frontière avec le Paraguay. }\end{array}$ & $\begin{array}{l}\text { Aucune; utiliser des mesures de } \\
\text { protection individuelle. }\end{array}$ & \multirow[b]{2}{*}{$\begin{array}{c}\text { Octobre à } \\
\text { mai }\end{array}$} & \multirow[b]{2}{*}{0} \\
\hline & & $\begin{array}{l}\text { Régions rurales des provinces du nord } \\
\text { de Jujuy et de Salta (le long de la } \\
\text { frontière avec la Bolivie). }\end{array}$ & $\begin{array}{l}\text { Chloroquine pour les séjours } \\
>2 \text { semaines; chloroquine ou } \\
\text { mesures de protection } \\
\text { individuelle uniquement pour les } \\
\text { séjours } \leq 2 \text { semaines. }\end{array}$ & & \\
\hline \multirow[t]{2}{*}{12} & \multirow[t]{2}{*}{ Brésil } & $\begin{array}{l}\text { Peu de transmission, voire aucune } \\
\text { transmission du paludisme aux chutes } \\
\text { d'Iguazu, dans la région de Pantanal, } \\
\text { dans les villes de Brasilia, de Recife, de } \\
\text { Rio de Janeiro, de São Paulo et de } \\
\text { Salvador, ou dans les autres régions } \\
\text { qui ne sont pas citées ci-dessous. }\end{array}$ & $\begin{array}{l}\text { Aucune; utiliser des mesures de } \\
\text { protection individuelle. }\end{array}$ & & \multirow[b]{2}{*}{15} \\
\hline & & $\begin{array}{l}\text { Régions jusqu'à } 900 \text { m d'altitude dans } \\
\text { la plupart des régions forestières des } \\
\text { États suivants : Acre, Amapá, } \\
\text { Amazonas, Rondônia, Roraima et } \\
\text { Tocantins (partie occidentale); et dans } \\
\text { certaines parties des États suivants : } \\
\text { Maranhāo (partie occidentale), Mato } \\
\text { Grosso (partie septentrionale), Pará } \\
\text { (sauf la ville de Belém) et Tocantins } \\
\text { (partie occidentale). La transmission se } \\
\text { produit également dans certaines } \\
\text { régions urbaines périphériques (Boa } \\
\text { Vista, Cruziero do Sul, Macapá, } \\
\text { Manaus, Maraba, Pôrto Velho, Rio } \\
\text { Branco et Santarém). }\end{array}$ & ATQ-PG, DOXY ou MFQ. & À l'année & \\
\hline \multirow[t]{3}{*}{13} & \multirow[t]{3}{*}{ Belize } & $\begin{array}{l}\text { Aucune transmission du paludisme à } \\
\text { Belize City et dans les îles fréquentées } \\
\text { par les touristes. }\end{array}$ & Aucune. & S.O. & S.O. \\
\hline & & $\begin{array}{l}\text { Risque faible dans les districts de } \\
\text { Belize, de Corozal et d'Orange Walk. }\end{array}$ & $\begin{array}{l}\text { Aucune; utiliser des mesures de } \\
\text { protection individuelle. }\end{array}$ & \multirow{2}{*}{ À l'année } & \multirow{2}{*}{0 à 5} \\
\hline & & $\begin{array}{l}\text { Risque modéré dans les districts de } \\
\text { Cayo, de Stann Creek et de Toledo. }\end{array}$ & Chloroquine. & & \\
\hline 14 & Équateur & $\begin{array}{l}\text { Aucune transmission du paludisme à } \\
\text { des altitudes supérieures à } 1500 \mathrm{~m}, \mathrm{y} \\
\text { compris à Cuenca, à Quito et dans } \\
\text { d'autres villes et villages des hautes } \\
\text { terres des Andes, ainsi que dans la ville } \\
\text { de Guayaquil et sur les îles Galápagos. }\end{array}$ & Aucune. & S.O. & S.O. \\
\hline
\end{tabular}




\begin{tabular}{|c|c|c|c|c|c|}
\hline & & $\begin{array}{l}\text { Toutes les autres régions jusqu'à } \\
1500 \text { m. Risque plus élevé le long du } \\
\text { littoral, dans le nord. }\end{array}$ & ATQ-PG, DOXY ou MFQ. & À l'année & 10 \\
\hline \multirow[t]{2}{*}{15} & \multirow[t]{2}{*}{ Colombie } & $\begin{array}{l}\text { Aucune transmission du paludisme : } \\
\text { dans les zones urbaines, y compris } \\
\text { Bogotá, sa région et Cartagena; à une } \\
\text { altitude supérieure à } 1600 \text { m; ou sur } \\
\text { les îles de l'archipel de San Andrés y } \\
\text { Providencia dans la mer des Caraïbes. }\end{array}$ & Aucune. & S.O. & S.O. \\
\hline & & $\begin{array}{l}\text { Zones rurales ou de jungle jusqu'à } \\
1600 \text { m d'altitude. }\end{array}$ & ATQ-PG, DOXY ou MFQ. & À l'année & 35 à 40 \\
\hline \multirow[t]{2}{*}{16} & \multirow[t]{2}{*}{ Guatemala } & $\begin{array}{l}\text { Aucune transmission du paludisme } \\
\text { dans les zones urbaines, dans les } \\
\text { régions à plus de } 1500 \text { m d'altitude ou } \\
\text { dans la ville de Guatemala, à Antigua } \\
\text { et au lac Atitlán. }\end{array}$ & Aucune. & S.O. & S.O. \\
\hline & & $\begin{array}{l}\text { Régions rurales jusqu'à } 1500 \text { m } \\
\text { d'altitude. }\end{array}$ & Chloroquine. & À l'année & 3 \\
\hline \multirow[t]{2}{*}{17} & \multirow[t]{2}{*}{ Honduras } & $\begin{array}{l}\text { Aucune transmission du paludisme } \\
\text { dans les villes de Tegucigalpa et de } \\
\text { San Pedro Sula. } \\
\text { Le risque est faible dans les régions } \\
\text { montagneuses à plus haute altitude } \\
\text { dans l'ouest, où des mesures de } \\
\text { protection individuelle peuvent être } \\
\text { envisagées. }\end{array}$ & Aucune. & S.O. & S.O. \\
\hline & & $\begin{array}{l}\text { Le risque est élevé dans les } \\
\text { départements de Gracias a Dios et des } \\
\text { Islas de la Bahia (Îles de la baie) et } \\
\text { modéré dans les départements } \\
\text { d'Atlantida, de Colon, d'Olancho et de } \\
\text { Yoro. }\end{array}$ & Chloroquine. & À l'année & 7 \\
\hline \multirow[t]{3}{*}{18} & \multirow[t]{3}{*}{ Vietnam } & $\begin{array}{l}\text { Aucune dans les zones urbaines, le } \\
\text { delta du Fleuve Rouge et la plaine } \\
\text { côtière du Vietnam central. } \\
\text { Rares cas dans le delta du Mékong. } \\
\text { En général, le parcours côtier souvent } \\
\text { emprunté entre Hô-Chi-Minh-Ville et } \\
\text { Hanoï avec des arrêts d'une nuit dans } \\
\text { des zones urbaines ne nécessite pas } \\
\text { de chimioprophylaxie. }\end{array}$ & $\begin{array}{l}\text { Utiliser des mesures de } \\
\text { protection individuelle. }\end{array}$ & \multirow{3}{*}{ À l'année } & \multirow{3}{*}{50 à 90} \\
\hline & & $\begin{array}{l}\text { Régions rurales, à l'exception de celles } \\
\text { énumérées ci-dessus. } \\
\text { Le risque est plus faible dans la ville de } \\
\text { Sa Pa, dans les montagnes au nord- } \\
\text { ouest d'Hanoï; des mesures de } \\
\text { protection individuelle peuvent être } \\
\text { envisagées pour les séjours inférieurs à } \\
1 \text { semaine, notamment pendant les } \\
\text { mois d'hiver. }\end{array}$ & ATQ-PG, DOXY ou MFQ. & & \\
\hline & & $\begin{array}{l}\text { Une résistance à la méfloquine a été } \\
\text { signalée dans la partie sud du pays, } \\
\text { dans les provinces suivantes : Dac Lac, } \\
\text { Gia Lai, Khanh Hoa (partie } \\
\text { occidentale), Kon Tum, Lam Dong, } \\
\text { Ninh Thuan (partie occidentale), Song } \\
\text { Be et Tay Ninh. }\end{array}$ & ATQ-PG ou DOXY. & & \\
\hline 19 & Cambodge & $\begin{array}{l}\text { Aucune transmission du paludisme } \\
\text { dans la ville de Phnom Penh ou dans la } \\
\text { région autour du lac Tonlé Sap (Siem } \\
\text { Reap). Transmission négligeable dans } \\
\text { les régions touristiques d'Angkor Wat et } \\
\text { de Siem Reap. }\end{array}$ & $\begin{array}{l}\text { Aucune; utiliser des mesures de } \\
\text { protection individuelle. }\end{array}$ & À l'année & 86 \\
\hline
\end{tabular}




\begin{tabular}{|c|c|c|c|c|c|}
\hline & & $\begin{array}{l}\text { Une résistance à la méfloquine est } \\
\text { signalée dans les provinces } \\
\text { occidentales de Banteay Meanchey, de } \\
\text { Battambang, de Koh Kong, d'Odder } \\
\text { Meanchey, de Pailin, de Kampot, de } \\
\text { PreahVihear, de Pursat et Siemreap (à } \\
\text { la frontière avec la Thaïlande). }\end{array}$ & ATQ-PG ou DOXY. & & \\
\hline & & Toutes les autres régions. & ATQ-PG, DOXY ou MFQ. & & \\
\hline \multirow[t]{3}{*}{20} & \multirow[t]{3}{*}{ Panama } & $\begin{array}{l}\text { Peu de transmission, voire aucune } \\
\text { transmission du paludisme à Panama } \\
\text { City, dans la zone du canal ou dans les } \\
\text { régions qui ne sont pas énumérées ci- } \\
\text { dessous. }\end{array}$ & $\begin{array}{l}\text { Aucune; utiliser des mesures de } \\
\text { protection individuelle. }\end{array}$ & \multirow{3}{*}{ À l'année } & \multirow{3}{*}{1} \\
\hline & & $\begin{array}{l}\text { Provinces et territoires indigènes } \\
\text { (comarques) le long du littoral des } \\
\text { Caraïbes et frontières avec le Costa } \\
\text { Rica et la Colombie : Bocas del Toro, } \\
\text { Chiriquí, Colón, Ngöbe-Buglé, Panamá } \\
\text { et Veraguas. }\end{array}$ & $\begin{array}{l}\text { Chloroquine pour les séjours } \\
>1 \text { semaine; chloroquine ou } \\
\text { mesures de protection } \\
\text { individuelle uniquement pour les } \\
\text { séjours < } 1 \text { semaine. }\end{array}$ & & \\
\hline & & $\begin{array}{l}\text { La plupart des transmissions se } \\
\text { produisent dans les provinces de l'est } \\
\text { du canal de Panama, vers la frontière } \\
\text { avec la Colombie. Une résistance de } P \text {. } \\
\text { falciparum à la chloroquine a été } \\
\text { signalée dans la province de Darién et } \\
\text { dans la comarque de Kuna Yala (San } \\
\text { Blas). }\end{array}$ & ATQ-PG, DOXY ou MFQ. & & \\
\hline 21 & Pakistan & $\begin{array}{l}\text { Toutes les régions jusqu'à } 2000 \mathrm{~m} \\
\text { d'altitude. Risque lié à } P \text {. vivax et à } P \text {. } \\
\text { falciparum. Le risque est plus faible } \\
\text { dans le nord, y compris à Islamabad, } \\
\text { surtout pendant les mois d'hiver en } \\
\text { raison des températures froides. }\end{array}$ & ATQ-PG, DOXY ou MFQ. & À l'année & 30 \\
\hline \multirow[t]{2}{*}{22} & \multirow[t]{2}{*}{ Kenya } & $\begin{array}{l}\text { Peu de transmission, voire aucune } \\
\text { transmission du paludisme à des } \\
\text { altitudes supérieures à } 2500 \mathrm{~m} \text { ou } \\
\text { dans la ville de Nairobi. }\end{array}$ & $\begin{array}{l}\text { Aucune; utiliser des mesures de } \\
\text { protection individuelle. }\end{array}$ & \multirow[t]{2}{*}{ À l'année } & \multirow[t]{2}{*}{85} \\
\hline & & $\begin{array}{l}\text { Toutes les régions jusqu'à } 2500 \mathrm{~m} \\
\text { d'altitude, sauf dans la ville de Nairobi. }\end{array}$ & ATQ-PG, DOXY ou MFQ. & & \\
\hline 23 & $\begin{array}{l}\text { Tanzanie } \\
\text { (République- } \\
\text { Unie de) }\end{array}$ & $\begin{array}{l}\text { Toutes les régions jusqu'à } 1800 \mathrm{~m} \\
\text { d'altitude. }\end{array}$ & ATQ-PG, DOXY ou MFQ. & À l'année & $>85$ \\
\hline \multirow[t]{2}{*}{24} & \multirow[t]{2}{*}{ Indonésie } & $\begin{array}{l}\text { Aucune transmission du paludisme } \\
\text { dans la municipalité de Jakarta, dans } \\
\text { les principales zones métropolitaines, y } \\
\text { compris Ubud, ou dans les principaux } \\
\text { centres de villégiature touristiques à } \\
\text { Bali et à Java. }\end{array}$ & Aucune. & S.O. & S.O. \\
\hline & & $\begin{array}{l}\text { En général, le risque est plus élevé } \\
\text { dans les régions plus orientales de } \\
\text { l'Indonésie : notamment dans les } \\
\text { provinces des Nusa Tenggara oriental, } \\
\text { des Moluques, des Moluques du Nord, } \\
\text { de la Papouasie (Irian Jaya) et la } \\
\text { Papouasie occidentale. Le risque existe } \\
\text { également sur l'île de Lombok et dans } \\
\text { les régions rurales de l'île Kalimantan } \\
\text { (Bornéo). Le risque de transmission est } \\
\text { faible dans les régions rurales de Java } \\
\text { et de Bali; des cas sporadiques ont été } \\
\text { signalés chez les voyageurs dans les } \\
\text { régions rurales de Bali. Dans les autres } \\
\text { parties du pays, le risque de paludisme } \\
\text { existe dans certains districts. }\end{array}$ & ATQ-PG, DOXY ou MFQ. & À l'année & 66 \\
\hline
\end{tabular}




\begin{tabular}{|c|l|l|l|}
\hline 25 & Nicaragua & $\begin{array}{l}\text { Peu de transmission, voire aucune } \\
\text { transmission du paludisme dans les } \\
\text { départements qui ne sont pas } \\
\text { énumérés ci-dessous. }\end{array}$ & $\begin{array}{l}\text { Aucune; utiliser des mesures de } \\
\text { protection individuelle. }\end{array}$ \\
\cline { 3 - 4 } & $\begin{array}{l}\text { Départements de Chinandega, de } \\
\text { Léon, de Managua et de Matagalpa, et } \\
\text { régions autonomes Atlántico Norte } \\
\text { (RAAN) et Atlántico Sur (RAAS). }\end{array}$ & Chloroquine. \\
\hline
\end{tabular}

La chimioprophylaxie n'est recommandée dans les régions à risque désignées que pendant la saison de transmission indiquée. La chimioprophylaxie doit toujours être utilisée en plus des mesures de protection individuelle.

ATQ-PG, atovaquone/proguanil; DOXY, doxycycline; MFQ, mefloquine 